Magdalena Kozak, PhD, https://orcid.org/0000-0002-2549-8007

Institut de Philosophie

Faculté de Philosophie

L'Academie Ignatianum de Cracovie

\title{
Le rôle et la signification de l'idée cartésienne de l'infini dans la métaphysique de Levinas ${ }^{1}$
}

\author{
Rola i znaczenie Kartezjańskiej idei Nieskończoności \\ w metafizyce Levinasa ${ }^{2}$
}

https://doi.org/10.34766/ fetr.v48i4.982

\begin{abstract}
This paper deals with the influence and significance of the Cartesian Idea of the Infinite on the philosophy of Emmanuel Levinas. The aim of this paper is to prove the thesis that it is impossible to understand Levinas's metaphysics without referring to Descartes's thought, especially in the aspect of the role and meaning of the idea of Infinity. Descartes' thinking is not the primary inspiration that Levinas admits to, however it is worth noting that the entire ethics of the Jewish thinker is built precisely on the concept of "infinity. " Therefore, at the beginning of the text I will briefly discuss the idea of Infinity as formulated by Descartes in the third Meditation, and then focus on analyzing the influence of this idea on Levinas' thought. The paper presented here will trace Levinas' philosophical path and the different moments and stages in the evolution of his thought, which would be difficult to understand in isolation from Descartes' idea of the Infinite. Both the establishment of a relationship with another person and with God are made possible by an absolute separation between the members of the relationship. Maintaining this separation, however, is made possible by transcendence, and thus central to the article: the infinity that separates the Self from the Other. The use of the idea of infinity in metaphysics/ethics enables Levinas to protect absolute otherness/difference from being absorbed into a unified and totalizing whole, from being neutralized by identity. Key concepts in Levinas's philosophy such as passivity, responsibility, substitution, proximity, and transcendence are founded precisely on the Cartesian notion of the Infinite. The text will conclude by outlining the doubts that may arise from a deeper analysis of Levinas's thought, as well as briefly juxtaposing the differences in understanding the idea of Infinity that clearly divide Descartes and Levinas. Both philosophers begin their philosophical journey from a common starting point: the solitary subject, going beyond himself, reaches the Infinite thereby overcoming his egoistic existence. However, in Descartes' case it happens on the way of intellectual effort of the cogitating Cogito and in Levinas's case on the way of offering oneself to the Other in an ethical relation. For Descartes discovers the idea of infinity within himself as an innate idea, while in Levinas this discovery comes from outside from the Other. Keywords: idea of Infinity, transcendence, Other, idea/ideatum, Descartes, Levinas, relation, passivity, responsibility, trauma

Résumé: L'article aborde la question de l'impact et de la signification de l'Idée cartésienne de l'infini sur la philosophie d'Emmanuel Levinas. Le but est de prouver la thèse selon laquelle il est impossible de comprendre la métaphysique de Levinas sans se référer à la pensée de Descartes, qui, bien que n'étant pas l'inspiration première reconnue par Levinas, construit toute son éthique sur la notion

\footnotetext{
${ }^{1}$ Article préparé grâce au programme "Excellent Science" (DNK/SN/466899/2020) du Ministère des Sciences et de l'Enseignement Supérieur en Pologne, dans le cadre du programme: "(Ir)pertinence de la phénoménologie? (Im)pertinence de la phénoménologie?"

${ }^{2}$ Artykuł w języku polskim dostępny jest na stronie:

https://www.stowarzyszeniefidesetratio.pl/Presentations0/2021-4Koza2.pdf
} 
d'infini. Dans la première partie du texte, nous discuteront brièvement l'idée de l'Infini telle que formulée par Descartes dans la troisième " Méditation », avant d'analyser son impact sur la pensée de Levinas. Nous retracerons le chemin philosophique de Levinas ainsi que divers moments et étapes de l'évolution de sa pensée, qui ne sauraient être compris qu'à l'aune de l'idée de l'Infini de Descartes. Seule une séparation absolue permet l'établissement d'une relation avec autrui et avec Dieu. Le maintien de cette séparation est pourtant possible grâce à la transcendance et, notion cruciale dans cet article, à l'infini qui sépare le Soi-même de l'Autre. Le recours à l'idée de l'infini en métaphysique/éthique permet à Levinas de protéger l'altérité/différence absolue contre l'absorption dans un tout homogène et totalisant, contre la neutralisation par l'identité. Les concepts clés de la philosophie de Levinas, tels que passivité, responsabilité, substitution, proximité et transcendance, sont fondés précisément sur la notion cartésienne d'Infini. En conclusion, nous exposeront les doutes susceptibles de surgir lors d'une analyse approfondie des pensées de Levinas et résumerons brièvement les différences dans la compréhension de l'idée de l'Infini qui divisent clairement Descartes et Levinas. Dans leurs cheminements philosophiques respectifs, les deux penseurs adoptent un point de départ commun: un sujet solitaire, en se dépassant soi-même, atteint l'Infini et brise ainsi son existence égoïste. Or, chez Descartes, cela se produit au prix d'un effort intellectuel accompli par le Cogito, alors que chez Levinas par le don de soi-même à l'Autre dans une relation éthique. Descartes découvre l'idée de l'infini en tant qu'idée innée; chez Levinas cette découverte vient du dehors - de l'Autre.

Mots-clés: idée de l'Infini, transcendance, Autre, idée/ideatum, Descartes, Levinas, relation, passivité, responsabilité, traumatisme

Abstrakt: W zaprezentowanym artykule zostaje podjęta kwestia wpływu i znaczenia Kartezjańskiej Idei Nieskończoności na filozofię Emmanuela Levinasa. Celem artykułu jest udowodnienie tezy, że nie da się zrozumieć metafizyki Levinasa bez sięgnięcia do myśli Kartezjusza, szczególnie w aspekcie roli i znaczenia idei Nieskończoności. Myślenie Kartezjusza nie jest pierwszoplanową inspiracją, do której przyznaje się Levinas, jednakże warto zauważyć, że cała etyka żydowskiego myśliciela jest zbudowana właśnie na pojęciu „nieskończoności”. Dlatego też na początku tekstu krótko omówię ideę Nieskończoności, sformułowaną przez Kartezjusza w trzeciej Medytacji, a następnie skupię się na analizie oddziaływania tej idei na myśl Levinasa. W przedstawionym artykule prześledzona zostanie droga filozoficzna Levinasa oraz różne momenty i etapy ewolucji jego myśli, które trudno byłoby zrozumieć w oderwaniu od idei Nieskończoności Kartezjusza. Zarówno zawiązanie relacji z drugim człowiekiem, jak i z Bogiem, są możliwe dzięki absolutnej separacji między członami relacji. Utrzymanie tej separacji jest jednak możliwe dzięki transcendencji, a więc kluczowej dla artykułu: nieskończoności, która oddziela Toż-Samego od Innego. Zastosowanie idei nieskończoności w metafizyce/etyce umożliwia Levinasowi ochronę absolutnej inności/różnicy przed wchłonięciem w jednolitą i totalizującą całość, przed zneutralizowaniem przez tożsamość. Kluczowe dla filozofii Levinasa pojęcia, takie jak bierność, odpowiedzialność, substytucja, bliskość, transcendencja są ufundowane właśnie na Kartezjańskim pojęciu Nieskończoności. W podsumowaniu tekstu zostaną przedstawione wątpliwości, które mogą pojawić się przy głębszej analizie myśli Levinasa, jak również krótko zostaną zestawione różnice $\mathrm{w}$ rozumieniu idei Nieskończoności, które w wyraźny sposób dzielą Kartezjusza i Levinasa. Obydwaj filozofowie zaczynają swoją drogę filozoficzną ze wspólnego punktu wyjścia: samotny podmiot, wychodząc poza siebie, dociera do Nieskończonego tym samym przełamując swoje egoistyczne istnienie. Jednakże u Kartezjusza dokonuje się to na drodze intelektualnego wysiłku poznającego Cogito a u Levinasa na drodze ofiarowania siebie Innemu $\mathrm{w}$ relacji etycznej. Kartezjusz odkrywa bowiem ideę nieskończoności w sobie jako ideę wrodzoną, a u Levinasa to odkrycie przychodzi z zewnątrz - od Innego.

Słowa klucze: idea Nieskończoności, transcendencja, Inny, idea/ideatum, Kartezjusz, Levinas, relacja, bierność, odpowiedzialność, trauma

\section{Introduction}


En analysant la philosophie d'Emmanuel Levinas dans le contexte de la pensée française contemporaine, il serait difficile de comprendre ses textes sans se référer aux racines modernes de sa philosophie. Il est intéressant de noter que parmi les nombreuses inspirations philosophiques clairement décelables dans les œuvres d'Emmanuel Levinas, la figure de Descartes n'apparaît pas au premier plan. Certes, Hegel, Husserl ou Heidegger ${ }^{3}$ ont eu une bien plus grande influence sur la formation de la pensée de ce philosophe français. Cependant, étant donné le statut de Descartes en tant que "père de la philosophie moderne » et son rôle dans la formation de l'ensemble de la tradition ultérieure de la pensée moderne dans la culture française ${ }^{4}$, il faut honnêtement admettre qu'il serait impossible de comprendre la métaphysique, et donc l'éthique de Levinas, sans faire référence à l'idée cartésienne de l'Infini. Cette idée, que nous souhaitons approfondir ici, est l'outil de base pour Levinas pour familiariser le lecteur avec le domaine de l'altérité totale et la condition du sujet qui s'éloigne de l'idée du Cogito solitaire ${ }^{5}$ au profit d'une relation personnelle avec l'Autre. Cette idée permet à Levinas de dépasser le niveau ontologique pour entrer dans la relation éthique, ce que nous tenterons de prouver dans cet article. L'Idée cartésienne de l'Infini se trouve sans doute au palmarès des images, souvent métaphoriques et symboliques, que Levinas aime utiliser pour mieux exprimer le sens de sa pensée.

Dans cet article, je voudrais présenter brièvement comment Levinas utilise l'idée cartésienne de l'Infini dans son concept de transcendance, qui fonde la relationnalité au sens large. Comme nous le verrons plus loin, en se référant à l'infini cartésien, Levinas en fait le fondement de la construction d'une relation métaphysique avec l'Autre: à la fois prochain et Dieu. L'idée de l'Infini empruntée à Descartes servira à Levinas à construire une subjectivité passive qui ne cesse d'exercer sa bonté et sa responsabilité sans limites. Il est extrêmement important dans cet exposé de montrer comment l'infini cartésien aide Levinas à exprimer la différence entre l'intérieur et l'extérieur et à maintenir le caractère absolu de la transcendance

\footnotetext{
${ }^{3}$ Dans le contexte de cet article, il parait important de rappeler que, dans ses commentaires, Levinas pointait Heidegger comme entièrement coupable de la réduction de l'infini et de la transcendance par l'enracinement du Dasein uniquement dans le domaine de l'être. Levinas qualifie l'existence heideggérienne de «païenne » et renchérit ainsi: «La philosophie de Heidegger est l'apogée de la pensée dans laquelle le fini ne renvoie pas à l'infini (...). L'ontologie heideggérienne subordonne le rapport avec l'Autre à la relation avec le Neutre » (Levinas, 2008, 178-179).

4 Un diagnostic intéressant de la philosophie française contemporaine est présenté par Jacek Migasiński qui désigne trois penseurs les plus marquants de ce cercle culturel ayant exercé la plus grande influence sur la formation de la philosophie française contemporaine. Montaigne, Pascal et Descartes sont les trois «pères fondateurs » sans lesquels il serait impossible de comprendre la pensée française contemporaine (cf. Migasiński, 2019, p. 12).

5 En analysant le rôle et l'influence de la pensée de Descartes sur la philosophie française contemporaine, Migasiński se concentre sur trois idées principales présentes chez le père de la philosophie moderne: l'idée de la méthode, l'idée du Cogito et l'idée de la nature illimitée de la volonté (liberté), encore que la troisième, évidemment liée à celle discutée dans cet article, dans l'interprétation de Migasiński ne peut pas être dissociée de l'idée du Cogito solitaire ; c'est sur ces trois idées que s'appuie le développement ultérieur de la métaphysique française (cf. Migasiński, 2019, p. 13).
} 
en dépassant l'immanent. J'ose supposer que l'idée cartésienne de l'Infini a permis à Levinas de construire une métaphysique de l'altérité absolue, qui fait exploser toute pensée et toute compréhension. "L'idée de l'infini ne surgit pas au sein du sujet pensant, elle signifie le franchissement des limites possibles de la subjectivité et, ainsi formulée, exprime la disproportionnalité fondamentale de l'idée de l'infini elle-même, dont elle est l'idée » (Migasinski, 2002, p. 18). Mettre en évidence le rôle et la signification de l'idée cartésienne de l'infini dans la pensée de Levinas vise à souligner une tendance claire présente dans la pensée française contemporaine qui, aux XXe et XXIe siècles, s'avise de transgresser ses positions initiales, tester les limites et tenter à répétition de dépasser sa propre légitimité. Un examen plus approfondi de l'importance de l'idée de l'infini dans la philosophie de Levinas permet de comprendre comment la phénoménologie excessive ${ }^{6}$ qui s'exerce actuellement recourt de plus en plus à la transgression et, à l'aide de moyens littéraires, souvent métaphoriques, tente d'exprimer ce qui échappe à nos capacités cognitives et linguistiques traditionnelles.

\section{L'idée de l'infini chez Descartes}

Tout d'abord, un extrait de la « Méditation troisième » de Descartes:

«Maintenant, c'est une chose manifeste par la lumière naturelle, qu'il doit y avoir pour le moins autant de réalité dans la cause efficiente et totale que dans son effet: car d'où est-ce que l'effet peut tirer sa réalité sinon de sa cause? et comment cette cause la lui pourraitelle communiquer, si elle ne l'avait en elle-même. Et de là il suit, non seulement que le néant ne saurait produire aucune chose, mais aussi que ce qui est plus parfait, c'est-à-dire qui contient en soi plus de réalité, ne peut être une suite et une dépendance du moins parfait. Et cette vérité n'est pas seulement claire et évidente dans les effets qui ont cette réalité que les philosophes appellent actuelle ou formelle, mais aussi dans les idées où l'on considère seulement la réalité qu'ils nomment objective » (Descartres, ouvrage en ligne).

Descartes dit que si j'ai en moi l'idée des choses extérieures, je ne peux pas dire avec certitude qu'elles existent pour de vrai, car je peux moi-même en être l'auteur. Et si je suis leur auteur, ces idées peuvent être de purs objets mentaux. Il en va autrement avec Dieu. Dieu, selon la définition de Descartes, est: « une certaine substance infinie et indépendante, dotée de la plus haute compréhension et puissance, qui m'a créé ainsi que tout ce qui existe » (Descartes, 2010, p. 50). Cette définition de Dieu implique Son existence réelle, car je - en tant que substance finie - ne peux, selon le principe de causalité, être à l'origine de l'idée d'une substance infinie, car je ne peux pas être l'auteur de quelque chose qui me dépasse

\footnotetext{
${ }^{6}$ François-David Sebbah, disciple et spécialiste de la philosophie de Levinas, écrit davantage sur ce sujet dans ses ouvrages dans lesquels il relève une tendance claire de la philosophie française contemporaine à l'autotransgression et à la mise à l'épreuve des limites de ses propres domaines (cf. Sebbah, 2001, p. 301-303 ; Sebbah, 2008, p. 186-189).
} 
éminemment. L'idée de l'Infini n'est pas comprise par moi comme une simple négation de la finitude car, comme précise Descartes: « au contraire, je comprends assez clairement qu'il y a plus de réalité dans une substance infinie que dans une finie, et que c'est donc en moi en quelque sorte en premier lieu une approche de l'infini avant le fini, c'est-à-dire de Dieu avant moi » (Descartes, 2010, p. 50). Puisque je suis un être fini, je ne peux pas être à l'origine de l'idée de l'Infini. Une idée comme effet requiert une cause qui se suffise à elle-même, et donc l'existence réelle de Dieu agissant en moi: c'est Lui qui m'a inculqué son idée du fait de son action. L'idée de l'Infini est donc finalement une idée innée.

\section{Reprise de l'idée de l'Infini par Levinas}

L'idée cartésienne de l'infini est pour Levinas l'outil de base pour montrer l'altérité totale, c'est une sorte de modèle qui exprime la différence absolue et la disproportion entre l'acte de penser et ce que l'on pense. Levinas doit à Descartes la conception selon laquelle l'idée de l'infini contient d'ores et déjà ce qui restera toujours extérieur à la pensée. L'idée de l'infini ne surgit pas au sein du sujet pensant, elle signifie le franchissement des limites possibles de la subjectivité et, ainsi comprise, exprime la disproportion fondamentale de l'idée et de l'infini lui-même dont elle est l'idée. L'idée de l'infini est la manière d'être l'infini (l'infinition), c'est-à-dire sa production au sein du sujet comme une sorte de révélation. Il serait erroné de croire que l'infini préexiste à sa révélation; sa nature infinie qui émerge dans le sujet sous forme de l'idée de l'extériorité absolue, d'un toucher de la transcendance, est en même temps la production de l'infini lui-même vis-à-vis du sujet donné.

En réfléchissant sur l'idée de Dieu, Descartes a esquissé le processus de pensée visant à briser le je pense. C'est Descartes qui a ouvert à Levinas la voie qui, en définitive, conduit à une rupture avec le Cogito. Dans l'interprétation de Descartes, ce que Levinas trouve important, ce n'est pas tant la preuve de l'existence de Dieu (en tant qu'être éminent, «superlatif de l'exister » et peut-être déjà dépassant l'ontologie), mais une sorte de " rupture de la conscience, qui n'est pas un refoulement dans l'inconscient mais un dégrisement ou un réveil secouant le 'sommeil dogmatique' qui se dort au fond de toute conscience reposant sur l'objet » (Levinas, 1994, p. 123). Dans un autre texte, Levinas la décrit comme la « rupture de la conscience, rupture qui n'est pas refoulement dans l'inconscient, mais dégrisement ou réveil » (Levinas, 2008, p. 259). De quoi s'agit-il? Le cogitatum transcende la cogitation, c'està-dire que l'idée même de Dieu transcende toute compréhension, va au-delà de toute réalité formelle, en annulant ainsi en quelque sorte la nature originelle de la conscience intentionnelle. Toute pensée, toute compréhension humaine, tout acte de désignation réduit l'ensemble des choses au domaine de l'existence, de la présence et de la représentation. Mais l'idée de Dieu fait voler cette pensée en éclats (cf. Levinas, 1994, p. 123). Cette «apport indépassable » - comme l'appelle Lévinas - montre qu'il n'y a pas d'idée de Dieu, dès lors 
que l'on dépasse l'ordre dans lequel l'idée conduirait à l'existence. L'idée de Dieu n'est que Dieu en moi. Ainsi, dans le sillage de Descartes, Levinas perçoit l'Infini qui ne peut être pensé, compris ou pénétré, car il va au-delà de ces processus. Il affirme, certes, que l'Infini habite la pensée, mais il s'agit d'un type d'habitation très différent de celui de l'inclusion du cogitatum dans la cogitation. "C'est une habitation passive et sans pareil parce que l'on ne peut pas la prendre sur soi » (Levinas, 1994, p. 124). Selon la nomenclature de Levinas, cette passivité qui dépasse toute passivité connue et compréhensible constitue l'essence de l'éveil.

Il semble nécessaire de bien distinguer, au sein de l'Idée de l'infini, une certaine « réalité formelle » et la "réalité objective ", qui constituent l'axe de la distinction radicale introduite par Descartes et suivie par Levinas. Levinas lui-même définit la " réalité formelle ${ }^{7}$ comme «L'Autre dans le même », ce qui signifie que ce «plus » (ideatum) est paradoxalement contenu dans ce "moins » (idée). Il est découle que l'infini ne peut être contenu dans la finitude, ce qui équivaudra pour Levinas à l'incapacité, pour le Soi-même, d'assimiler l'Autre. Or, c'est précisément cette altérité radicale, qui échappe à toutes les possibilités cognitives et linguistiques, qui m'éveille et m'ouvre à la recherche de l'Autre. Un autre aspect est la «réalité objective » qui signifie l'Infini lui-même. Ce n'est pas la représentation que l'on s'en fait, l'idée que le sujet s'en façonne dans son esprit, l'idée qui l'éveille, le provoque et le fait éclater de l'intérieur, mais l'Infini lui-même. Cette distinction exprime une certaine incompatibilité de l'idée et de l'ideatum, l'échappement absolu de l'Infinité aux limites de l'idée elle-même. ${ }^{8}$ C'est cette insuffisance que Levinas appellera « pensée " qui pense plus qu'elle ne pense. Ce concept, peut-être vague et peu précis d'un point de vue logique, devient plus compréhensible si l'on se souvient que la relation entre l'idée de l'infini et son ideatum est d'ordre éthique (métaphysique) et non pas cognitif. Dans sa propre interprétation de cet ideatum tiré de Descartes, Levinas verra le Bien qui vient à l'homme précisément de la dimension de l'infini et qui est plus fondamental et primordial qu'une simple action de penser. Par conséquent, l'innovation de Levinas par rapport à l'idée cartésienne de l'Infini consiste à atteindre la dimension du Bien que cet Infini ouvre. Et c'est précisément le rapport entre l'idée et l'ideatum tiré de Descartes qui nous permet de maintenir la transcendance absolue du Bien, qui dépasse nos représentations de celui-ci ainsi que nos capacités linguistiques et cognitives.

Dans la troisième Méditation de Descartes, Levinas décrypte le constat selon lequel l'infini est en quelque sorte antérieur, en moi, au concept de finitude, c'est-à-dire que l'idée de Dieu précède l'idée de moi-même. La rupture avec l'actualité du Cogito par le biais de

7 Ce sujet est discuté plus en détail dans le commentaire de Tadeusz Gadacz sur Levinas. En se référant à la distinction précitée des deux aspects de l'Idée de l'infini, il dit que c'est dans l'aspect formel l'infini stimule le fini, ce qui est dérangeant et inquiétant au point de faire éclater l'identité et interdire tout repos (cf. Gadacz, 1990, p. 264).

8 Tadeusz Gadacz écrit dans ce contexte: « Le transcendent est lui-même un ideatum. Il est infiniment éloigné de l'idée. La distance qui sépare l'ideatum de l'idée de cet ideatum est le contenu de l'ideatum lui-même » (cf. Gadacz, 1990, p. 266). 
l'idée de l'Infini en nous se traduit par une passivité absolue et radicale qui ne laisse aucune place pour la réceptivité, mais dans laquelle il y a de la place pour une souffrance sans bornes (Levinas, 1994, p. 125). Comment faut-il comprendre cela? L'idée de l'Infini est déposée en nous. En suivant Descartes, Levinas préconise que l'idée d'Infini nous soit « inculquée ». Cette inoculation, qui exigeait auparavant une passivité absolue, se traduit par une surprise ou une sensibilité à quelque chose auquel l'on ne peut résister, par une ouverture et un éveil radicaux. "Il estime que le fait de nous implanter une idée insaisissable brise cette présence à soi-même, qui est la conscience, force les barrières et les contrôles, renverse l'exigence d'accepter ou d'assimiler tout ce qui vient du dehors> (Levinas, 1994, p. 125).

Le moment de la négation semble ici extrêmement important. L'idée de l'Infini en moi ne peut pas être comprise comme le résultat d'une négation formelle et logique du fini. Une telle compréhension serait certainement insuffisante et ne correspondrait pas à ce qu'écrit Descartes. Levinas joue avec le mot «infini ». L'In-finitum peut et doit être compris non seulement comme «non » (négation), mais aussi " en » (en moi). "La naissance de la négativité n'a pas lieu dans la subjectivité qui nie, mais dans l'idée même de l'infini, c'est-àdire dans la subjectivité comme idée de l'infini »(Levinas, 2008, p. 260). L'infini ne signifie pas seulement la négation, mais l'infini en moi. C'est exactement la raison pour laquelle l'infini déchire le fini. ${ }^{9}$ L'idée de l'infini permet la subjectivité elle-même car elle m'a été inculquée (mise en moi) au moment de ma création. La réalité du Cogito est interrompue par ce qui est inimaginable, ce qui ne peut être intentionnellement pensé.

C'est donc une idée an-archique, venue à l'homme d'un au-delà du temps, d'un passé plus lointain que tout ce qui est révolu, accessible seulement comme trace dans le visage de l'autre. Cet aspect de la chronologie semble ici important car c'est précisément l'inadéquation entre l'antérieur et le postérieur qui permet l'existence d'une relation métaphysique. L'ideatum est antérieur à l'idée. L'infini précède le fini. Ainsi l'ideatum transcende l'idée parce qu'il se situe aussi loin dans le passé, est si distant du présent, qu'il est impossible de percevoir sa relation avec l'idée comme un tout. La primordialité absolue de l'Infini signifie sa transcendance, son échappement au fini, l'ici et maintenant, son insaisissabilité. L'idée de l'Infini ne peut donc pas venir du Soi, du sujet pensant lui-même, et elle ne vient pas d'un besoin qui pourrait être satisfait. Elle ne peut qu'éveiller un désir qui ne trouve jamais son accomplissement, car sa nature est d'éveiller constamment le sujet ${ }^{10}$, de le déranger et

\footnotetext{
${ }^{9}$ L'on voit ici une nette inspiration par Descartes, pour qui l'Idée de l'Infini n'est pas une simple négation de la finitude. Descartes lui-même admet: « (...) je vois manifestement qu'il se rencontre plus de réalité dans la substance infinie que dans la substance finie, et partant que j'ai en quelque façon premièrement en moi la notion de l'infini, que du fini » (Descartes, ouvrage en ligne).

${ }^{10} \mathrm{Il}$ convient de se référer à la description de cette situation livrée par Jacek Migasiński qui écrit, notamment: «L'idée de l'Infini ne vient pas du 'je' et ne surgit pas d'un besoin du 'je'. Le mouvement vient ici de l'être pensant et non pas d'un sujet pensant (...). L'Infini n'est donc pas un 'objet' de la
} 
d'appeler à plus qu'être, à penser plus que l'on ne pense. C'est une idée qui s'épuise à se révéler, à se montrer, qui ne tire pas son sens de l'acte de se manifester. Le contact avec cette idée nous éveille, éveille notre conscience, en devenant en pratique une obligation et un sens qui enjoint quelque chose. Ce moment où l'idée de l'infini vient s'inculquer en moi, qualifié par Levinas de traumatisme, est crucial pour le façonnement ultérieur de la subjectivité.

\section{L'idée de l'infini dans la relation avec un autre humain}

Ce choc (le traumatisme), que je ne peux contrôler, causé par l'instillation de l'Infini dans le fini, se produit lorsque je me retrouve face à face avec un autre être humain. C'est le moment qui montre clairement que l'idée cartésienne de l'Infini ouvre la voie à Levinas pour construire sa propre métaphysique, comprise comme éthique. L'événement de la rencontre avec l'Autre est marqué (affection) par la présence de l'Infini dans le visage de l'Autre qui se tient devant moi et donne un sens éthique à notre rencontre, en nous emmenant au-delà de l'être et m'appelant à prendre la responsabilité pour lui. L'incapacité de saisir l'Infini par la pensée engendre ma passivité absolue en tant que destinataire de cet Infini. L'intrigue du sens liée à l'idée de l'infini fait que l'infini dépasse et transcende radicalement l'événement de la rencontre avec l'Autre. Levinas décrit ce moment comme "un réveil douloureux », « l'éblouissement où l'œil tient plus qu'il ne tient ; ignition de la peau qui touche et ne touche pas ce qui, par-delà le saisissable, brûle »(Levinas, 1994, p. 128), ce qui m'oblige à une attitude de passivité plus passive que toute passivité antérieure. C'est sur ce terrain que la négativité du «In » de l'Infiniutum fait naître un désir qui ne peut pas être satisfait. ${ }^{11}$ C'est le désir de l'Infini, qui se nourrit de sa propre faim et qui « s'exalte en tant que désir, et qui s'éloigne de la satisfaction à mesure qu'il s'approche du désirable » (Levinas, 1994, p. 129). Ce désir dépasse le domaine de l'ontologie, ne définit pas son but ou sa fin (par opposition à un besoin), et ne trouve jamais son accomplissement. Le désir de l'infini, qui s'éveille en moi dans la relation avec l'Autre, lorsque je rencontre son visage qui m'appelle, est essentiellement le désir du Bien compris comme altruisme, acte de se donner à l'autre et de répondre sans cesse à l'appel qui jaillit de son visage. A ce propos, Levinas n'a pas peur de se référer au terme «Amour » et écrit que «L'amour n'est possible que par l'idée de l'Infini -

cognition, à la mesure d'un regard contemplatif, mais le Désiré qui éveille le Désir, c'est-à-dire qui se révèle à la pensée qui à chaque instant 'pense plus qu' elle ne pense' » (Cf Migasiński, 2002, p. 21).

${ }^{11}$ Dans la philosophie de Levinas, le désir s'oppose au besoin. Alors que le besoin appartient à l'ordre du Même (Soi-Même) et peut être satisfait, le désir naît dans l'ordre de la transcendance, dans le domaine du différent. C'est l'Idée de l'Infini qui me donne accès au désir qui ne trouvera jamais son accomplissement. Comme le souligne Forthomme, le besoin ne peut être traité comme une pure négation, et le désir comme quelque chose de positif. " Le désir métaphysique est la pauvreté de la richesse ou l'insuffisance de la plénitude. En effet, ce désir ne peut naître que chez celui qui n'a plus besoin ; c'est un besoin de luxe qui naît de moi-même ou d'une totalité satisfaite. Et ce, même si le désir métaphysique ne peut en aucun cas naître à partir du Même repu, sans l'appel de l'Extériorité » (Fothomme, 1979, p. 183). 
par l'Infini mis en en moi, par le 'plus' qui dévaste et réveille le 'moins' » (Levinas, 1994, p. 129). Afin que le Désir de l'Infini ne retourne pas dans la sphère de l'immanence et ne s'y referme pas, il doit constamment dépasser l'être, le transcender sans cesse pour sauver son altruisme. Ce qui est désiré (le Bien, Dieu) doit être séparé au sein du désir - comme ce qui est encore désiré, inatteignable, mais proche grâce aux traces laissées dans le visage de l'Autre. C'est pourquoi le Bien me renvoie à un autre être humain, et la relation éthique avec lui devient l'espace où se révèle l'Infini. C'est l'éveil dont parle Levinas, l'éveil à la proximité, à la bienveillance, à la responsabilité pour l'Autre. L'infini me rend totalement passif face à l'autre, fait de moi son otage, qui, avant même d'avoir entendu l'appel, est déjà fidèle à un engagement qu'il n'a pas pris dans sa liberté, mais qui lui a été assigné dans un temps anarchique.

Dans «Totalité et infini », Levinas utilise l'idée de l'Infini de Descartes pour séparer l'extérieur de l'intérieur et ainsi préserver la nature transcendante du Désir. Il parle « d'un désir parfaitement désintéressé - la bonté et précise que le désir et la bonté présupposent une relation concrète »(Levinas, 2002, p. 41). L'idée cartésienne de l'infini sert ici à Levinas pour décrire l'épiphanie du visage, pour transmettre son expression et sa signification. « Le visage $\mathrm{d}^{\prime}$ Autrui détruit à tout moment, et déborde l'image plastique qu'il me laisse, l'idée à ma mesure et à la mesure de son ideatum - l'idée adéquate » (Levinas, 2002, p. 42). "L'idée de l'Infini (...) se produit concrètement comme une relation avec le visage. Et seule l'idée de l'Infini préserve l'extériorité de l'Autre par rapport au Soi-même, malgré cette relation » (Levinas, 2002, p. 229). L'idée cartésienne de l'Infini permet aussi à Levinas de sauvegarder l'altérité absolue de l'Autre rencontré dans l'événement de la rencontre. Il estime que «l'Autre reste infiniment transcendant, infiniment étranger - mais son visage, survenant comme une épiphanie et m'appelant, se détache du monde «(Levinas, 2002, p. 227).

Au fil du temps, cependant, l'idée cartésienne de l'Infini prend une expression encore plus profonde lorsque, dans ses écrits « tardifs » (au stade de " Autrement qu'être ou au-delà de l'essence), Levinas l'utilise et s'en inspire pour construire la subjectivité dans un sens sacrificiel. Il présente le sens éthique de l'Infini à partir de ma relation avec mon prochain et de ma responsabilité à son égard. La passivité de la subjectivité n'est pas une pure abstraction, mais elle consiste en pratique à faire éclater la présence immanente et prend la forme d'une responsabilité de plus en plus radicale pour l'Autre. La responsabilité qui ne cesse de croitre à mesure que je l'assume signifie une rupture totale avec ma liberté et celle de l'Autre. Ma responsabilité - contre moi - parce que c'est la manière dont l'Autre me dérange ou s'impose à moi, c'est-à-dire qu'il est proche de moi, consiste à écouter ou à entendre ce cri. C'est un réveil. «La proximité du prochain, c'est ma responsabilité pour lui: approcher, c'est être gardien de son frère et être gardien de son frère, c'est être son otage » (Levinas, 1994, p. 135). L'infini, à partir duquel l'Autre me parle, brise mon identité et fait de moi un otage, me place dans la posture de l'un pour l'autre, dans la promptitude de m'offrir 
pour mon prochain. L'infini, qui me marque par l'appel du visage de l'Autre ${ }^{12}$, me rend infiniment responsable de mon frère, au-delà de tout contrat, au-delà de tout présent, audelà de toute liberté, car j'ai été nommé par le Bien à ce désintéressement. L'ideatum du Bien infini dépasse à tous égards l'idée du Bien infini que j'assume. L'ideatum de la culpabilité, de la responsabilité et du sacrifice surpasse en tout point la culpabilité, la responsabilité et le sacrifice que j'endosse et que je suis prêt à exprimer. C'est pourquoi la responsabilité pour l'Autre brise mon identité de l'intérieur et ne me permet en aucun cas de me constituer dans l'unité et la cohérence d'un tout fermé. Dès lors, mon existence consiste à me dépasser continuellement lorsque je me donne sans cesse à l'Autre. «Responsabilité qui ne laisse pas de temps: sans présent de recueillement ou de rentrée en soi ; et qui me met en retard: devant le prochain, je comparais plutôt que je n' apparais. Je réponds d'emblée à une assignation. Déjà le caillouteux noyau de ma substance est dénoyauté. Mais la responsabilité à laquelle je suis exposé dans une telle passivité ne me saisit pas comme une chose interchangeable , car personne ici ne peut se substituer à moi ; en en appelant à moi comme à un accusé qui ne saura récuser l'accusation, elle m'oblige comme irremplaçable et unique. Comme élu. Dans la mesure où elle en appelle à ma responsabilité, elle m'interdit tout remplaçant. Irremplaçable pour la responsabilité, je ne peux sans carence ou sans faute ou sans complexe, me dérober au visage du prochain: me voici voué à l'autre sans démission possible »(Levinas, 1994, pp. 134- 135). Sans aucun doute, la responsabilité est la quintessence de l'infini chez Levinas. La responsabilité est absolument infinie, constitue une vocation sans limites, qui me place dans une position de l'un pour l'autre, c'est-à-dire de substitution. La responsabilité sans fin conduit à l'idée de se donner pour autrui, ce qui pour Levinas est le summum de l'héroïsme éthique. En ce sens, l'infini me conduit à la perfection éthique, pratiquement assimilée au suicide moral.

\section{4) L'idée de l'infini dans la relation avec Dieu}

Le schéma formel emprunté à Descartes, utilisé pour exprimer la relation métaphysique entre le Même et l'Autre, trouve son application non seulement dans la relation avec l'Autre entendu comme autrui, mais aussi avec l'Autre entendu comme Dieu. L'idée de l'infini permet à Levinas de préserver non seulement l'altérité absolue de l'autre, mais aussi l'altérité et la transcendance absolues du Tiers. L'on voit à nouveau que l'idée de

12 Il est à noter ici que, partant de l'idée cartésienne de l'Infini, Levinas montre la transcendance de l'Autre par rapport au Soi-même, et donc la paradoxalité de la situation dans laquelle ce qui est le plus extérieur, le plus éloigné de moi, m'émeut le plus et m'éveille à l'ouverture éthique. Migasiński caractérise le paradoxe de cette situation en évoquant " un sujet agité, éthiquement sensible, enclin à répondre à la 'supplique' du prochain. La 'séparation' radicale entre moi et la 'dimension du divin' se transforme en une 'proximité' radicale du rapport social. La transcendance 'se produit' dans une relation éthique et nous place au-delà des catégories de l'être » (cf. Migasiński, 2017, p. 183). 
l'Infini dépasse nos capacités de réflexion et cognitives, en s'orientant vers des relations de nature personnelle, où les délibérations théoriques sur Dieu sont remplacées par le désir de l'Infini, l'admiration et l'émerveillement ${ }^{13}$. La distance infranchissable entre l'idée et l'ideatum chez Descartes se reflète dans la notion de séparation, qui s'applique à la fois à la relation avec autrui et avec Dieu. Dieu est si infiniment différent de moi que je n'ai aucune possibilité de L'embrasser avec ma raison, ma connaissance, mes pensées. Il est à l'abri de toutes mes tentatives de L'absorber ou de Le réduire au plan du même (de l'identité). J'ai son idée en moi, pourtant l'Infini lui-même dépasse infiniment son idée. ${ }^{14}$ Selon Levinas, l'idée de l'Infini exige que le Même et l'Autre soient simultanément en relation et se libèrent de cette relation, tout en restant absolument séparés (Levinas, 2002). Levinas rejette une fois de plus la métaphysique de l'unité et du tout, optant pour la métaphysique de l'altérité et de l'infini. En rejetant la position classique de nombreux philosophes (par exemple, Parménide, Plotin, Spinoza) qui, dans leurs systèmes, poursuivaient l'idée de l'unité et de l'union avec ce qui fut le premier, Lévinas voit la nécessité de rompre avec cette tradition afin de sauver la vraie transcendance. L'on peut remarquer que Platon l'y aide avec son idée du Bien, qui s'inscrit parfaitement dans le modèle formel d'idée-ideatum de Descartes et complète le concept de désir métaphysique. L'existence du Bien absolu et infini qui appelle l'homme et se fait désirer par lui le plus ardemment est la quintessence de la relation métaphysique avec Dieu. Alors que Plotin voit tout comme venant de l'Un et étant une émanation au sein d'un tout, Platon adopte une position contraire: le bien se situe est au-dessus de tout et est si radicalement différent de ce qui est connu qu'il ne satisfera aucun besoin, mais suscitera un désir toujours plus profond. En coupant avant la tradition plotinienne du tout englobant, Levinas utilise l'Idée de l'Infini de Descartes pour briser la compréhension totalisante de la relation métaphysique; il opte pour Dieu comme Celui qui ne suscite pas un besoin, mais uniquement le désir. ${ }^{15}$

${ }^{13}$ Il est intéressant que Descartes suive un chemin similaire pour atteindre le Créateur. Partant de la certitude du Cogito (qui, chez Levinas, correspond au Soi-même égoïste immergé dans le monde des besoins, de la jouissance et du plaisir), il atteint l'Infini, qui ne peut être que contemplé et admiré (chez Levinas aussi, l'Infini entendu comme quintessence de l'altérité transcende le fini et sape tout ce qu'il m'était connu auparavant). Descartes écrit dans la « Méditation troisième »: « (...) Il me semble très à propos de m'arrêter quelque temps à la contemplation de ce Dieu tout parfait, de peser tout à loisir ses merveilleux attributs, de considérer, d'admirer et d'adorer l'incomparable beauté de cette immense lumière, au moins autant que la force de mon esprit, qui en demeure en quelque sorte ébloui, me le pourra permettre » (Decartes, ouvrage en ligne).

${ }^{14} \mathrm{Il}$ convient de rappeler ici le commentaire de Marek Jędraszewski, qui discute de l'application de la structure formelle cartésienne dans la pensée de Levinas, en soulignant l'importance de la séparation. Jędraszewski dit: "Il y a une vraie séparation entre Dieu et l'homme: tout peut être porté à la conscience sauf Dieu, Il existe vraiment séparé du sujet, oui, Il en est le fondement. De manière négative, l'on peut dire que, dans cette relation, celui qui contient n'absorbe pas l'objet contenu, car je ne peux pas contenir l'Infini, et l'objet contenu n'absorbe pas celui qu'il contient, car je suis séparé de l'Infini » (Jędraszewski, 1990, p. 115).

15 Dans ce contexte, Jędraszewski écrit sur la compréhension négative par Levinas de la relation métaphysique, qui abandonne l'optique de l'unité, du tout et de l'identité basée sur le principe de participation. « La participation signifie, selon Levinas, une telle relation de l'homme avec Dieu, qui 
Les analyses qui précèdent font surgir des doutes et des questions suivantes qui en découlent: Comment peut-on désirer quelqu'un qui est absolument différent de soi? De quelle manière et par quel biais cet Autre me « touche » et me " réveille » s'il est absolument différent? Où se trouve ce point/cet espace commun qui nous permet de nouer des relations, bien que nous soyons absolument séparés? Pourquoi le sujet nourrit-il le désir qu'il ne pourra jamais satisfaire? Quel est le rôle de l'idée de l'infini dans le fait que le désir est sans cesse approfondi et que le sujet ne se décourage pas à désirer de plus en plus? Voici les questions qui révèlent certaines lacunes et déficits dans la construction de Levinas et obligent en même temps le lecteur à chercher plus loin.

\section{Conclusion}

En résumé: sans aucun doute, la structure formelle cartésienne fondée sur l'incompatibilité de l'idée avec l'ideatum a servi à Levinas de modèle de sa propre structure métaphysique à travers laquelle il a voulu exprimer la relation entre Soi et l'Autre. L'idée de l'Infini, empruntée à Descartes, a permis à Levinas de maintenir la transcendance de l'Autre par rapport au Soi-même et de la sauver des velléités totalisantes de ce dernier. Descartes est parti de la certitude du Cogito pour ensuite arriver à l'Infini. Un chemin similaire est parcouru par Levinas, pour qui l'éloignement et la distance (la séparation) sont les moments nécessaires pour rencontrer l'Autre. "Dans l'idée de l'infini on pense ce qui est toujours extérieur aux pensées » (Levinas, 2010, p. 8) et il ajoute, dans la même veine, que la distance qui sépare l'ideatum de l'idée détermine le contenu de l'ideatum lui-même. L'infini appartient à l'être transcendant en tant que transcendant, l'infini est ce qui est absolument différent (Levinas, 2002, p. 39). Le sujet ne peut donc jamais comprendre l'Infini - il ne peut contenir que son idée, car c'est en cela que consiste la vraie transcendance: elle réside dans la différence radicale entre une idée et son ideatum. Du fait de la différence radicale entre l'idée et l'ideatum empruntée à Descartes, Levinas maintient une véritable transcendance. Alors que Descartes croyait que le sujet, par sa conscience connaissante et ses capacités intellectuelles, pouvait dominer toutes les idées sauf l'idée de Dieu, le sujet levinassien, lui aussi, peut dominer et régner sur toute altérité, mais ne peut pas atteindre ce pouvoir sur l'Autre. Cet Autre est une transcendance absolue qui échappe à mes tendances impérieuses, qui ne me laisse que son idée.

Cependant, outre les similitudes dans la compréhension de l'Infini chez Descartes et Levinas discutées ci-haut, il convient de mentionner, en conclusion, quelques différences importantes dans les conceptions des deux philosophes. Dans ses « Méditations », Descartes est parvenu au constat de l'existence de Dieu en analysant l'idée de l'Infini qu'il portait en lui

est typique de la religion, où une personne s'immerge dans le sacré de manière inconsciente, où elle succombe aux émotions et même à l'extase, où elle s'expose au viol subi du fait de ce sacré » (Jędraszewski, 1990, p. 123). 
comme idée innée. Ainsi, l'idée de l'Infini apparaît chez Descartes au sein du sujet qui la découvre comme objet de sa spéculation. La position de Levinas est différente: l'idée de l'infini me vient du dehors, de l'Autre, comme absolument autre et différente de moi ; cela conduit Lévinas à croire que l'existence de Dieu échappe à tous les processus intellectuels et n'exige en fait aucune preuve. Il en est ainsi car Levinas accorde la primauté à la relation éthique, alors que Descartes, à la relation cognitive. Cela engendre a une conséquence sérieuse: chez Descartes, la métaphysique classique occupe l'une des places les plus importantes, et l'éthique se situe loin en arrière-plan. Levinas, en enrichissant et développant l'Idée de l'Infini empruntée à Descartes, ouvre une nouvelle voie qui doit mener plus loin, là où Descartes n'est pas parvenu. La rencontre avec l'Autre (et surtout avec son visage appelant) permet à Levinas de reconnaître la précellence de l'éthique sur tous les autres domaines de la philosophie et, de plus, de l'identifier à la métaphysique. C'est là, face à l'Autre qui m'appelle depuis la dimension de l'Infini, que surgissent les traces de Dieu. Selon Levinas, l'éthique, au-delà de la vision et de la certitude, dessine la structure de l'extérieur comme tel. La morale n'est pas une branche de la philosophie, mais la philosophie première (Levinas, 2002, p. 367). Ainsi, l'ouvrage « Tout et l'infini » de Levinas, qui se révèle comme une réflexion sur la prima philosophia assimilée à l'éthique, revendique le droit de se substituer aux «Meditationes de prima philosophia» cartésiennes. Les traces de Dieu, imprimées dans le visage de l'Autre, montrent un ordre autre que celui de l'être. C'est un ordre qui ne provient pas de ce monde, se situe au-delà de l'être, exprime une transcendance absolue inaccessible à la pensée et à la cognition humaine, mais une transcendance qui éveille la sensibilité, fait appel à l'humanité, milite pour l'humanisme. Cet infini ne peut être compris ni par la raison ni par l'empirisme, mais peut être vécu comme la profondeur de l'expérience, comme ma passivité face à l'Autre. ${ }^{16}$ Levinas affirme que tel est le sens d'une trace. Le visage est dans la trace de l'Absent, la seule ouverture où le sens du Transcendant n'invalide pas le transcendant. L'existence qui laisse une trace signifie passer, partir, se libérer du monde (Levinas, 2008, pp. 209-210). ${ }^{17}$

Enfin, le dernier point mérite d'être souligné. L'on voit que le sujet de Descartes et le sujet de Levinas commencent leur existence au même point: le Cogito de Descartes est solitaire jusqu'à ce qu'il en arrive à l'Idée de l'Infini en lui-même et le Soi-même de Levinas est une hypostase solitaire condamnée à prendre soin de lui-même et à rester dans la sphère des besoin de l'existence égoïste. L'affranchissement du domaine de l'ego solitaire ou égoïste

${ }^{17}$ En analysant la passivité ouverte à la lecture des traces de l'Infini, Migasiński attire l'attention sur un nouvel ordre: non seulement l'au-delà de l'être, mais aussi l'ordre du non-être. Une telle compréhension de l'infini le libère de l'idée et le transfère au niveau de l'ouverture éthique, c'est-à-dire de la passivité face à l'Autre. «Être en mode de laisser des traces, de passer et de partir, c'est en fait ne pas être. Ainsi comprise, la transcendance/l'infini ne peut être une 'idée', elle/il ne peut afficher aucune similitude avec des êtres 'de ce monde' » (Cf. Migasiński, 2017, p. 188). 
s'accomplit par la découverte de la transcendance.18 Le Cogito solitaire découvre dans son esprit l'idée de l'infini et, partant, le principe de son existence qui est le Dieu infini. Cela lui ouvre la voie à de nouvelles possibilités, dépassant sa propre subjectivité, plus loin et plus haut. Chez Levinas, le cheminement est similaire: le Soi-même, auparavant concentré uniquement sur la satisfaction de ses besoins liés à l'existence, ne découvre qu'au moment de la rencontre avec l'Autre la relationnalité qui contredit sa vie antérieure. Il s'avère que à l'instar de Descartes - l'idée de l'infini parlant à travers le visage de l'Autre me révèle une dimension nouvelle, différente: la transcendance qui étale devant moi une nouvelle perspective, radicalement différente, celle de l'exister autrement. Par conséquent, l'on peut risquer d'affirmer que dans le cas des deux philosophes, l'idée de l'infini donne le sens ultime à l'existence humaine. Levinas résume ainsi le sens ultime de l'idée cartésienne de l'Infini en disant qu'il doit avoir l'idée d'infini, que Descartes croit être aussi une idée de perfection, afin de connaître sa propre imperfection (Levinas, 2008 , p. 185). Ainsi, la dépendance du sujet de l'Infini est une évidence pour les deux philosophes. L'existence du sujet n'a de sens que grâce à la référence à ce qui est transcende le monde du fini.

L'on peut aussi remarquer, revenant au thème annoncé dans l'introduction de cet article, que l'idée de l'infini introduite dans la pensée moderne par Descartes et redécouverte par Levinas prouve l'existence d'un besoin fondamental, dans la philosophie française, de dépassement, de transgression et transcendance au sens large. ${ }^{19}$ La volonté toujours présente d'exprimer l'impossible, de dépasser le cadre et les systèmes actuels, apparaît chez de nombreux penseurs français. Une critique claire de la philosophie intellectuelle, de la pensée scientifique et de la recherche constructiviste se reflète, par exemple, dans la philosophie de la vie et dans l'aversion, largement répandue dans la philosophie française, pour la pensée systémique et spéculative prégnante dans la philosophie allemande. Le motif de l'idée de l'Infini empruntée au père de la pensée française moderne, servant de fil conducteur à cet article, montre l'enracinement ancien, dans la pensée française, du désir de franchir et de surmonter les barrières, que la découverte par Descartes de l'espace de l'infini permet de réaliser jusqu'à ce jour.

\section{Bibliographie:}

Adamiak, M. (2008). O kobiecie, która nawiedza myśl. Kobieta jako figura inności w koncepcji podmiotu Emmanuela Levinasa, Warszawa: Wydawnictwa Akademickie i Profesjonalne.

18 En analysant la conception de l'infini chez Descartes, Marzena Adamiak écrit sur le dépassement par le sujet de son ego, le franchissant des limites liées à la sphère de l'être, sur les possibilités supplémentaires de transcendance et de transgression (Cf. Adamiak, 2007, p. 53-55).

19 Jacek Migasiński retrace brillamment la genèse de la condition de la philosophie française contemporaine, en soulignant le rôle de G. Bataille, P. Klossowski, M. Blanchot, M. Foucault, J. Derrida et G. Deleuze dans la formation de la philosophie française contemporaine (cf. Migasiński, 2017, p. 175-180 et Migasiński, 2019, p. 11-17). 
Descartes, R. (2010). Méditations métaphysiques, ouvrage en ligne, http://abu.cnam.fr/cgibin/donner_html?medit3.

Forthomme, B. (1979). Un philosophie de la Transcendance: la métaphysique d'Emmanuel Levinas, Paris: La pensée Universelle.

Gadacz, T. (1990). Wolność a odpowiedzialność. Rosenzweiga i Levinasa krytyka Heglowskiej wolności ducha, Kraków: Wydawnictwo Naukowe Papieskiej Akademii Teologicznej.

Jędraszewski, M. (1990). Wobec Innego. Relacje międzypodmiotowe w filozofii Emmanuela Levinasa, Poznań: Wydawnictwo UAM.

Migasiński, J. (2019). W strone fenomenologii nie zjawiskowej, Warszawa: PWN.

Migasiński, J. (2017), Levinas: filozofia opętania?, Przegląd Filozoficzny - Nowa Seria, 2 (102), 175-193.

Levinas, E. (1994). De Dieu qui vient à l'idée, trad. [en polonais] M. Kowalska, Kraków: Znak.

Levinas, E. (2002). Totalité et infini, trad. [en polonais] M. Kowalska, Warszawa: PWN.

Levinas, E. (2008a). Dieu, la mort et le temps, trad. [en polonais] J. Margański, Kraków: Znak.

Levinas, E. (2008b). En découvrant l'existence avec Husserl et Heidegger, trad. [en polonais] E. Sowa, Warszawa: Wydawnictwo IFiS PAN.

Sebbah, F-D. (2001). L'épreuve de la limite. Derrida, Henry, Levinas et la phénoménologie, Paris: PUF.

Sebbah, F-D, Salanskis J.-M. (2008). Usage contemporains de la phénoménologie, Paris: Ed. Sens\&Tonka. 